\title{
Teorías subjetivas de profesores sobre gestión del tiempo instruccional y clima de aula
}

\author{
Miguel A. Alarcón-Alvial' ${ }^{1}$, Cristián R. Oyanadel ${ }^{1,2}{ }^{*}$, Pablo J. Castro-Carrasco ${ }^{3,4}$, e Ingrid N. González ${ }^{3}$ \\ (1) Universidad de Concepción, Facultad de Ciencias Sociales, Dpto. de Psicología, Concepción, Chile \\ (correo-e: miguealarcon@udec.cl; coyanadel@udec.cl) \\ (2) Universidad de Granada, Centro de Investigación Mente, Cerebro y Comportamiento (CIMCYC), Grupo CTS-261, \\ Granada, España. \\ (3) Universidad de La Serena, Facultad de Humanidades, Dpto. de Psicología, Coquimbo-Chile. \\ (correo-e: pablocastro@userena.cl : igonzalez@userena.cl) \\ (4) Universidad Católica del Maule, Talca-Maule, Chile.
}

${ }^{*}$ Autor a quien debe ser dirigida la correspondencia

Recibido Mar. 30, 2020; Aceptado Jun. 1, 2020; Versión final Jul. 29, 2020, Publicado Oct. 2020

\begin{abstract}
Resumen
La presente investigación tuvo como objetivo reconstruir las teorías subjetivas (TS) del clima de aula y tiempo instruccional de 24 docentes de establecimientos de grupo socioeconómico bajo. Los datos fueron recolectados mediante encuesta sociodemográfica y entrevista episódica semiestructurada, y luego analizados utilizando procedimientos de la teoría fundamentada. Como hallazgos, se identificaron TS sobre facilitadores de la gestión del tiempo, como el buen comportamiento del estudiante, y el compromiso del docente. Por otro lado, obstaculizadores de la gestión del tiempo instruccional, como disposición desfavorable del estudiante hacia el aprendizaje, improvisación de la clase por parte del docente, y particularidades desfavorables de establecimientos de grupo socioeconómico bajo. La gestión del clima de aula se percibe como un elemento que permite mejorar el rendimiento y comportamiento de los estudiantes. Se concluye que existen distintos factores que pueden afectar la gestión del tiempo instruccional como el comportamiento adecuado y la disciplina del estudiante y el compromiso y cercanía por parte del docente.
\end{abstract}

Palabras clave: teorías subjetivas; tiempo; clima de aula; gestión pedagógica; relación estudiante-profesor

\section{Teacher's subjective theories on instructional time management and classroom environment}

\begin{abstract}
The objective of this research study was to reconstruct the subjective theories (ST) of the classroom environment and the instructional time of 24 teachers from low-income schools. Data collection methods included a sociodemographic survey and semi-structured episodic interviews. Collected data were analyzed using grounded theory procedures. The results showed ST facilitators of time management, such as good student behavior and teacher engagement. Instructional time management obstacles included low student drive and interest in learning, teacher improvisation in the classroom, and unfavorable features found in lowincome schools. Classroom environment management is perceived as an element that allows students to improve their performance and behavior. It is concluded that instructional time management can be affected by factors such as student behavior and discipline and teacher engagement and approachability.
\end{abstract}

Keywords: subjective theories; time; classroom environment; pedagogical management; teacher-student relationships 


\section{INTRODUCCIÓN}

El uso del tiempo es uno de los factores que más afectan la cultura y la gestión pedagógica, impactando en cómo se regula la relación profesor-estudiante en los procesos de enseñanza y aprendizaje (Martinic, 2015; Ugwulashi, 2012). Con respecto a lo último, se ha encontrado que en Chile, los docentes tienen dificultades para gestionar el tiempo instruccional, dedicando escasos minutos para introducir y cerrar una clase (Martinic et al., 2013). La gestión del tiempo en el aula presenta una gran importancia, ya que influye en el rendimiento académico de los estudiantes, lo cual implica una mayor eficiencia en el rol pedagógico al gestionar el tiempo instruccional de manera efectiva (Aronson et al., 1998). Por el contrario, aquellos docentes con una gestión no adecuada suelen desarrollar acciones ineficaces y poco dirigidas, lo que podría llevar a desperdiciar recursos materiales y humanos (Kayode y Ayodele, 2015)

El tiempo dedicado a la relación profesor-estudiante y a la gestión del ambiente de aula es el mejor predictor de un buen desempeño escolar, por ejemplo, el desarrollar estrategias enfocadas en establecer reglas y propiciar un entorno estructurado permitirían una instrucción adecuada de la clase (Lopes et al., 2017). Aun cuando se establece el clima de aula como un factor relevante para el éxito académico de los estudiantes y su bienestar (Wang y Degol, 2016), muchos establecimientos educacionales continúan enfocándose sólo en mejorar aspectos curriculares, a través de la mejora en rendimientos en evaluaciones, recursos académicos y desarrollo profesional de los docentes, ignorando con ello el papel que juega el clima de aula (Cohen et al., 2009).

Actualmente existen investigaciones que abordan la gestión pedagógica y del tiempo de los docentes en aula, las que en su mayoría corresponden a análisis de pautas de observación (semi o completamente estructuradas) por evaluadores externos, en directo o video (Strasser et al., 2009), sin presentar o visibilizar la perspectiva de los profesores, la cual cumple un papel fundamental en la generación de conocimiento, actitudes, valores y habilidades de niños, niñas y adolescentes (Pérez et al., 2015). Con base en lo anterior, y con el fin de aportar desde el significado de estos agentes educativos, la presente investigación tiene por objetivo reconstruir las teorías subjetivas (TS) de los docentes sobre la gestión del tiempo y del clima de aula.

\section{OTROS ANTECEDENTES}

El tiempo como constructo mental en la cotidianeidad es un hecho objetivo y lineal, que permite organizar la realidad social en estados temporales (pasado, presente y futuro). Sin embargo, el tiempo es vivenciado subjetivamente, y a través de componentes cognitivos, motivacionales y sociales (Boniwell y Zimbardo, 2004), se otorga significado y sentido a la experiencia humana. Con respecto al ámbito educacional y al cómo se gestiona el tiempo en las aulas, surge el concepto de Tiempo Instruccional (TI), el cual se define como una herramienta de mejora educativa con la cual se fomenta el uso óptimo del tiempo en el aula. En este sentido, la gestión $\mathrm{TI}$ es definida como aquellas prácticas que los docentes realizan para administrar el tiempo de enseñanza, con el fin de transmitir los conocimientos académicos de manera adecuada (Ugwulashi, 2013).

La gestión del TI es un proceso que implica la jerarquización de necesidades y la priorización de tareas dentro del aula (Khan et al., 2016), lo que permitiría optimizar los recursos disponibles y gestionar adecuadamente los conflictos en el aula (Ugwulashi, 2013). Si bien es una actividad que regularmente realizan los docentes, esto no es algo sencillo, puesto que el tiempo no es algo material que se pueda manejar con facilidad (Khan et al., 2016), debido a las múltiples demandas emergentes a las que se ven enfrentados los docentes en el aula, además no existen espacios donde se enseñe a los docentes o estudiantes a tomar conciencia sobre la importancia de manejar el tiempo adecuadamente (Álvarez y Lobo, 2020).

Considerando estos antecedentes, se pueden dilucidar ciertas consecuencias en relación al uso del tiempo en la planificación del trabajo en aula, encontrándose problemas en la gestión del TI, en cuanto se ha hallado que los docentes utilizan casi un $72 \%$ para el desarrollo de la clase y el resto para el inicio y cierre. Este problema, si bien fue investigado bajo el Sistema de Evaluación del Desempeño Profesional Docente (o Evaluación Docente), no logra adecuar las acciones de los profesores para mejorar la gestión del TI, puesto que muchos dedican mucho tiempo para el desarrollo, no realizando un inicio adecuado y tampoco anticipando un cierre a sus clases. Además, se ha visto que el mayor tiempo de uso en el desarrollo es utilizado por los estudiantes para realizar sus trabajos de forma individual, autogestionando ellos mismos las actividades a realizar durante el desarrollo de la clase (Martinic et al., 2013), lo que puede dificultar el proceso de aprendizaje debido a la falta de dirección por parte del docente.

Los docentes tienden a externalizar las causas en los problemas de gestión del aula, ya sea a nivel de clima de aula o del uso del tiempo, dando poco énfasis a la responsabilidad de lo que realizan y sucede en la sala de clases. En este sentido, es importante que los agentes educativos identifiquen aquellas actitudes y comportamientos que impactan negativamente en la gestión pedagógica, aún más cuando se trabaja con una población de estudiantes que provienen de contextos socioeconómicos bajos o de contextos familiares con 
altos índices de conflictos psicosociales (Nail et al., 2012), quienes presentarían mayores dificultades a nivel de gestión del clima de aula y del TI, presumiblemente por las dificultades en el control de la disciplina y del orden en la sala de clases (Ascorra et al., 2016; Martinic et al., 2013).

Para investigar la perspectiva de los docentes se recurre a dilucidar las Teorías Subjetivas (TS) que tienen éstos sobre el tema. Las TS son un conjunto de ideas, opiniones y argumentos que los profesores utilizan para justificar sus decisiones educacionales (Cuadra-Martínez et al., 2017). Estas se asemejan a explicaciones de teorías científicas, ya que poseen una estructura argumentativa hipotética, con la cual pretenden explicar los fenómenos vividos, presentando un elevado nivel de coherencia (Flick 2006). Esta estructura argumentativa que puede ser expresada verbalmente, no es meramente contemplativa, sino que es una manifestación de la proactividad humana, por cuanto puede llevar una persona a actuar o por el contrario, inhibir una conducta. Esto quiere decir que el comportamiento del profesor, o la intencionalidad de la acción de este, está mediada en parte por las TS que el docente tenga (Catalán, 2016).

El impacto en la investigación de TS en docentes, a través de la entrevista episódica como instrumento de recolección de datos, implica un punto de partida para apoyar el cambio en la práctica docente. Este cambio implica la modificación de estructuras y contenidos de estas representaciones, las cuales permiten desarrollar una forma más compleja de interpretar la realidad, una mayor conciencia respecto a la autoeficacia en el cambio o control de los fenómenos, y la generación de nuevas relaciones entre constructos subjetivos que pueden dar origen a nuevas TS, caracterizadas también por una mayor complejidad (Catalán, 2016; CuadraMartínez y Castro-Carrasco, 2018). La pertinencia de utilizar entrevistas episódicas y la relación con las TS, radica en que este tipo de entrevista, permite la indagación de aspectos biográficos derivados de la experiencia propia del docente, por cuanto dichos aspectos actúan como fuentes de información para la posterior reconstrucción de TS (Catalán, 2016).

La importancia del estudio de las TS en contextos educativos, es que al extraer experiencias de los docentes, el profesor se enfrenta a la posibilidad de monitorear su propia práctica en aula a través del relato provocando con ello el desarrollo de actitudes reflexivas que mejorarían la práctica docente (Bonilla, Castro-Carrasco y Gómez, 2020). En base a lo anterior, la presente investigación tuvo como objetivo reconstruir las TS del clima de aula y tiempo instruccional en docentes de establecimientos de grupo socioeconómico bajo.

\section{MÉTODO}

Para abordar el propósito de este estudio se realizó una investigación cualitativa descriptiva interpretativa, con el fin de acceder con profundidad a las TS que tienen los docentes acerca del problema planteado.

\section{Selección de participantes}

Participaron 24 docentes de establecimientos educacionales de grupo socioeconómico bajo (GSE bajo) de tres regiones de Chile (Atacama, Coquimbo y Biobío). Los establecimientos de GSE bajo fueron clasificados como tal según datos del Ministerio de Educación. En cada región participaron 8 docentes que abarcaran las características de experiencia, género y ciclo en el cual el docente realiza clases, las cuales se detallan en Tabla 1.

Tabla 1. Distribución de participantes

\begin{tabular}{|l|l|c|c|c|c|c|c|}
\hline & & \multicolumn{2}{|c|}{ Atacama } & \multicolumn{2}{c|}{ Coquimbo } & \multicolumn{2}{c|}{ Bio-bío } \\
\cline { 2 - 9 } & Experiencia & Mujer & Hombre & Mujer & Hombre & Mujer & Hombre \\
\hline \multirow{2}{*}{ Enseñanza Básica } & Más de 15 años & 1 & 1 & 1 & 1 & 1 & 1 \\
\cline { 2 - 9 } & Menos de 15 años & 1 & 1 & 1 & 1 & 1 & 1 \\
\hline \multirow{2}{*}{ Enseñanza Media } & Más de 15 años & 1 & 1 & 1 & 1 & 1 & 1 \\
\cline { 2 - 9 } & Menos de 15 años & 1 & 1 & 1 & 1 & 1 & 1 \\
\hline & Total & \multicolumn{3}{|c|}{8} & \multicolumn{2}{|c|}{8} \\
\hline
\end{tabular}

Instrumentos de recolección de datos

Para la obtención de información se utilizó un cuestionario sociodemográfico que permitió identificar criterios como la experiencia, edad, escolaridad y ciclo en el que se desempeñaban como docentes. Además, se utilizaron entrevistas episódicas, con el fin de recabar y reconstruir TS individuales, indagando sobre el tiempo instruccional y clima de aula. La entrevista episódica posibilita la producción de representaciones asociadas al contexto de las personas, puesto que se encuentra más próxima a la experiencia del sujeto y al entorno que la produjo, más que otras formas de recolección de información (Flick, 2006). Generando un guion con 
temas sobre 1) Explicaciones subjetivas acerca del tiempo en la escuela; 2) Explicaciones subjetivas acerca del tiempo escolar en relación con la institución educativa; 3 ) Explicaciones subjetivas acerca del rol docente en relación al tiempo en la escuela. Las entrevistas fueron grabadas en audio y posteriormente transcritas y analizadas. Todos los docentes firmaron voluntariamente un consentimiento informado, aprobado por el Comité Ético-Científico de la Universidad de La Serena.

\section{Procedimiento de análisis}

Se utilizó parte de la teoría fundamentada (Strauss y Corbin, 1998) para abordar el procedimiento de categorías conceptuales, donde los códigos obtenidos se contrastaron mediante el método de comparación constante hasta la codificación axial, a fin de plantear preguntas, descubrir propiedades y dimensiones que se encuentran implícitas en los datos a través de las categorías y subcategorías generadas en la codificación abierta (Strauss y Corbin, 1998). El proceso de codificación fue realizado mediante el software ATLAS.ti 7 Scientific Software Development.

\section{RESULTADOS}

Se muestran los resultados en tablas, ordenados por categorías y subcategorías generadas para toda la muestra, los cuales fueron agrupados en familias de códigos relativas a TS que otorgaban, por un lado, explicaciones a condiciones que obstaculizan y/o facilitan la gestión del tiempo instruccional. Por otro lado, se agruparon las TS sobre los beneficios que conlleva la gestión del clima de aula, y sobre las consecuencias negativas que produce la no gestión del mismo, asociadas también a cómo estas afectarían positiva o negativamente la gestión del tiempo instruccional. Por último, se describe un esquema (Figura 1) que permite organizar las TS en función de sus relaciones.

Tabla 2: Categoría, Obstaculizadores de la gestión del tiempo instruccional y Subcategoría, Obstaculizadores del estudiante.

\begin{tabular}{|c|c|c|}
\hline № & Teoría subjetiva & Comentario \\
\hline 1.1 & $\begin{array}{l}\text { Si los estudiantes se } \\
\text { distraen, el docente } \\
\text { deberá destinar } \\
\text { tiempo de instrucción } \\
\text { para retomar su } \\
\text { atención. }\end{array}$ & $\begin{array}{l}\text { Las TS asociadas a los obstaculizadores por parte del estudiante, se interponen a las } \\
\text { planificaciones que los docentes realizan a sus clases, puesto que si aparecen en el } \\
\text { aula el profesor debe reorientar su accionar pedagógico y cambiar lo anteriormente } \\
\text { planificado. Estos cambios hacen que los docentes "pierdan el control del tiempo" } \\
\text { destinado a cada una de las actividades de la clase: ... "es el profesor el que tiene } \\
\text { que lograr sacarlos de ahí, para llevarlos a la concentración, y uno no considera esos } \\
\text { tiempos (destinados a captar nuevamente la atención del estudiante)" (Profesor } \\
\text { enseñanza media, más de } 15 \text { años de experiencia). Además de las distracciones por } \\
\text { parte de los estudiantes, desde la perspectiva de los profesores, existen también } \\
\text { aquellos que no presentan una buena actitud hacia el aprendizaje y, por lo tanto, } \\
\text { retrasan el quehacer pedagógico: "... Un curso que no tiene, que no quiere trabajar, } \\
\text { no quiere hacer nada, no le gusta el ramo, no le gusta el profesor, no le gustan los } \\
\text { contenidos, imposible hacerle clase a ese curso o es muy difícil." (Profesor enseñanza } \\
\text { media, menos de } 15 \text { años de experiencia). }\end{array}$ \\
\hline 1.2 & $\begin{array}{l}\text { Si los estudiantes } \\
\text { provienen de familias } \\
\text { de situación } \\
\text { vulnerable, entonces } \\
\text { se deberá destinar } \\
\text { tiempo para suplir } \\
\text { necesidades } \\
\text { afectivas, dejando } \\
\text { menos tiempo para la } \\
\text { instrucción } \\
\text { académica. }\end{array}$ & $\begin{array}{l}\text { También se encuentran aquellas características que son de origen familiar de los } \\
\text { estudiantes, mostrándose que desde la perspectiva de los docentes, si los estudiantes } \\
\text { provienen de familias en situación de vulnerabilidad, los docentes deberán utilizar } \\
\text { parte del tiempo en aula para suplir ciertas necesidades afectivas de sus estudiantes, } \\
\text { dejando la instrucción académica en un segundo plano “... tú ahí eres psicólogo, } \\
\text { sociólogo, asistente social, tú cumples todos esos roles, y por eso, esos tiempos a ti } \\
\text { lamentablemente no te alcanzan, porque tú lo tienes que ver. Tienes que tener una } \\
\text { parte afectiva muy grande con ellos, la carencia afectiva ahí es muy fuerte” (Profesor } \\
\text { enseñanza básica, menos de } 15 \text { años de experiencia). }\end{array}$ \\
\hline 1.3 & $\begin{array}{l}\text { Si los estudiantes no } \\
\text { respetan al docente, } \\
\text { entonces este deberá } \\
\text { destinar tiempo para } \\
\text { establecer la relación } \\
\text { de autoridad en el } \\
\text { aula. }\end{array}$ & $\begin{array}{l}\text { Si bien se manifiesta que presentar un buen clima de aula, favorece una buena } \\
\text { gestión del tiempo por parte del docente, la relación también se da a la inversa. Por lo } \\
\text { tanto, los docentes tienen la TS según la cual la existencia de un clima de aula hostil } \\
\text { perjudicaría la gestión del tiempo realizada por el docente, más aún cuando los } \\
\text { estudiantes no respetan al profesor: “... Entonces uno tiene que inmediatamente, } \\
\text { quizás perder más tiempo de clase para que ellos vean que no, que el profesor es una } \\
\text { autoridad dentro de la sala de clases y que él dicta las normas" (Profesor enseñanza } \\
\text { media, más de } 15 \text { años de experiencia). }\end{array}$ \\
\hline
\end{tabular}


Tabla 2: continuación.

\begin{tabular}{|c|c|c|}
\hline 1.4 & $\begin{array}{l}\text { Si los estudiantes } \\
\text { llevan sus problemas } \\
\text { personales y } \\
\text { familiares al aula, } \\
\text { entonces el docente } \\
\text { dedica tiempo de la } \\
\text { instrucción } \\
\text { académica para } \\
\text { contener } \\
\text { emocionalmente. }\end{array}$ & $\begin{array}{l}\text { Dadas las características personales de los estudiantes, quienes tienden a llegar a los } \\
\text { establecimientos con problemas familiares, los docentes sienten que son } \\
\text { responsables por el bienestar psicológico de sus estudiantes, y por lo tanto dedican } \\
\text { tiempo de sus clases para conversar con ellos y aconsejarlos. Debido a que los } \\
\text { docentes conocen a sus estudiantes, se dan cuenta cuando tienen problemas, y en su } \\
\text { rol como formadores buscan ayudarlos siempre que pueden, aunque están } \\
\text { conscientes que el tiempo no les da abasto para realizar las labores de contención } \\
\text { emocional y las respectivas clases. Los docentes consideran que es necesario apoyar } \\
\text { a los estudiantes emocionalmente, independiente de que esto afecte el tiempo } \\
\text { disponible para la clase, puesto que estos problemas impactan negativamente en los } \\
\text { aprendizajes y rendimiento académico: “...porque tú ves eso, ves esa necesidad, que } \\
\text { el propio niño te está dando a conocer, entonces uno, obviamente le nace esa } \\
\text { inquietud de saber, y si tú puedes ayudarlo, lo haces, entonces, claro... entonces el } \\
\text { tiempo que le dedicaba a ellos, claro era en mi clase” (Profesor enseñanza básica, } \\
\text { menos de } 15 \text { años de experiencia). }\end{array}$ \\
\hline
\end{tabular}

Tabla 3: Categoría, Obstaculizadores de la gestión del tiempo instruccional y Subcategoría, Obstaculizadores del establecimiento.

\begin{tabular}{|c|c|c|}
\hline № & Teoría subjetiva & Comentario \\
\hline 2.1 & $\begin{array}{l}\text { Si el clima de aula es } \\
\text { negativo, el docente } \\
\text { deberá postergar sus } \\
\text { actividades de } \\
\text { instrucción para } \\
\text { resolver problemas, } \\
\text { dejando menos } \\
\text { tiempo para la clase. }\end{array}$ & $\begin{array}{l}\text { Existen situaciones derivadas del clima de aula, donde se considera que si bien es } \\
\text { necesario intervenir cuando ocurren situaciones problemáticas entre los alumnos, } \\
\text { estas intervenciones influyen en la gestión del tiempo de aula, ya que en ciertas } \\
\text { ocasiones no se logra cumplir con lo planificado. La gestión de la convivencia escolar } \\
\text { por parte de los docentes se tiende a realizar en dos escenarios, una ocurre dentro de } \\
\text { la misma clase y que por ende afecta la cantidad de tiempo que queda para continuar } \\
\text { con la instrucción académica, y otro escenario, es durante los momentos cuando el } \\
\text { docente se encuentra en sus horas no lectivas, donde este debe dejar de lado sus } \\
\text { labores de planificación: "yo podría determinar un tiempo para algo y surgen mil cosas } \\
\text { que lo hacen irreal, ejemplo tenemos planificado realizar una reunión... atravieso el } \\
\text { pasillo y se agarraron a combos, insultaron al profesor" (Profesor enseñanza media, } \\
\text { más de } 15 \text { años de experiencia). }\end{array}$ \\
\hline 2.2 & $\begin{array}{l}\text { Si tienes muchos } \\
\text { estudiantes en el } \\
\text { aula, entonces } \\
\text { gestionar el tiempo } \\
\text { será más difícil. }\end{array}$ & $\begin{array}{l}\text { Algunas de las explicaciones de los docentes, de por qué afecta el número de } \\
\text { alumnos, es porque al ser muchos estudiantes, no puedes dedicarles el tiempo } \\
\text { necesario a todos, por lo que muchos estudiantes quedarán sin ser supervisados, y } \\
\text { esto puede hacer que queden rezagados con sus aprendizajes: “...Si incide (cantidad } \\
\text { de alumnos por sala), porque el profesor nunca va a tener la total visión y me ha } \\
\text { pasado varias veces en todos mis años de mi trayectoria, de que un niño a veces, no } \\
\text { se da cuenta que no está aprendiendo” (Profesor enseñanza básica, más de } 15 \text { años } \\
\text { de experiencia). }\end{array}$ \\
\hline 2.3 & $\begin{array}{l}\text { Si existe diversidad } \\
\text { de estudiantes, } \\
\text { entonces el docente } \\
\text { debe detenerse para } \\
\text { que todos aprendan. }\end{array}$ & $\begin{array}{l}\text { Los profesores consideran que el tener distintos tipos de estudiantes en el aula, con } \\
\text { respecto a distintos ritmos de aprendizaje, necesidades educativas especiales, o tipos } \\
\text { de atención y concentración, afecta en cómo el docente gestiona su tiempo en aula: } \\
\text { “... yo no puedo pasar a otro contenido o a otra unidad si ese grupo no entendió } \\
\text { entonces me detengo, me detengo, me detengo, hasta que aprendan y ahí se pasa el } \\
\text { tiempo" (Profesor enseñanza básica, menos de } 15 \text { años de experiencia). }\end{array}$ \\
\hline
\end{tabular}

Tabla 4: Categoría, Obstaculizadores de la gestión del tiempo instruccional y Subcategoría, Obstaculizadores del docente.

\begin{tabular}{|l|l|l|}
\hline № & Teoría subjetiva & Comentario \\
\hline 3.1 & $\begin{array}{l}\text { Si tienes jefatura a } \\
\text { cargo tendrás menos } \\
\text { tiempo para planificar } \\
\text { adecuadamente las } \\
\text { clases. }\end{array}$ & $\begin{array}{l}\text { El hecho de que ciertos docentes tengan a cargo la jefatura de un curso, hace que } \\
\text { estos tengan menor tiempo disponible para planificar sus clases, puesto que deben } \\
\text { realizar otras labores administrativas alusivas al curso y cada uno de sus estudiantes, } \\
\text { entre las que se encuentran atención de apoderados, gestión de la convivencia } \\
\text { escolar, preparación de reuniones, y atender situaciones emergentes que ocurren con } \\
\text { sus estudiantes, entre otras. }\end{array}$ \\
\hline 3.2 & $\begin{array}{l}\text { Si el tiempo se } \\
\text { gestiona de forma } \\
\text { inadecuada, } \\
\text { entonces se pierde } \\
\text { más tiempo de } \\
\text { instrucción. }\end{array}$ & $\begin{array}{l}\text { Con respecto a las consecuencias que tiene una gestión del tiempo inadecuada por } \\
\text { parte del docente, se encuentra que se afecta a sí mismo, es decir, si el docente no } \\
\text { gestiona adecuadamente el tiempo, va a tener como consecuencia perder más tiempo } \\
\text { en poder hacer su clase. Sin embargo, se plantea que, para evitar perder el tiempo, el } \\
\text { docente debe planificar adecuadamente su clase, y de ninguna forma realizar clases } \\
\text { de forma improvisada, puesto que de esta forma el docente no podrá tener el manejo } \\
\text { de los tiempos destinados a cada parte de esta: “...bueno va a depender de como uno } \\
\text { Ileve planificada la clase, si yo llevo una clase improvisada eh... no voy a tener } \\
\text { manejo de los tiempos, cuando yo llevo una clase planificada, uno va aprendiendo a } \\
\text { manejar los tiempos y una cuando conoce también el contexto y los alumnos" } \\
\text { (Profesor enseñanza media, más de 15 años de experiencia). }\end{array}$ \\
\hline
\end{tabular}


Tabla 5: Categoría, Facilitadores de la gestión del tiempo instruccional y Subcategoría, Facilitadores por parte del docente.

\begin{tabular}{|l|l|l|}
\hline № & Teoría subjetiva & Comentario \\
\hline 1.1 & $\begin{array}{l}\text { Si conoces a los } \\
\text { estudiantes, entonces } \\
\text { puedes gestionar el } \\
\text { tiempo de acuerdo a } \\
\text { sus necesidades de } \\
\text { aprendizaje. }\end{array}$ & $\begin{array}{l}\text { La cercanía con los estudiantes, vista como la relación afectiva entre el docente y sus } \\
\text { estudiantes favorece la gestión del tiempo del profesor. Esto se origina luego de que } \\
\text { estructuras de la clase, con el fin de alinear las expectativas, intereses y motivaciones } \\
\text { de los estudiantes: ...Sé cuándo están cansados, por ejemplo. Eso te permite } \\
\text { cambiar inmediatamente de estrategia o cambiar de lo que estás haciendo.... } \\
\text { Entonces ¿qué se crea mas que el lazo afectivo? que pa mi es súper importante, se } \\
\text { crea esto que tu podi manejar el tiempo " (Profesor enseñanza básica, más de 15 } \\
\text { años de experiencia). }\end{array}$ \\
\hline 1.2 & $\begin{array}{l}\text { Si el docente es } \\
\text { comprometido, } \\
\text { entonces pondrá más } \\
\text { esfuerzos en mejorar } \\
\text { su gestión } \\
\text { pedagógica. }\end{array}$ & $\begin{array}{l}\text { un profesor tiene "vocación” para la docencia, entonces pondrá mayor esfuerzo en } \\
\text { aplicar una adecuada gestión del tiempo en sus clases: “...Se puede ver en la } \\
\text { vocación que tiene el docente, eso termina impactando a la organización de la } \\
\text { escuela, en los resultados, en los aprendizajes...” (Profesor enseñanza media, más } \\
\text { de 15 años de experiencia). }\end{array}$ \\
\hline $\begin{array}{l}\text { Si el docente tiene } \\
\text { más experiencia, } \\
\text { entonces su gestión } \\
\text { del tiempo será } \\
\text { mejor. }\end{array}$ & $\begin{array}{l}\text { La experiencia que tenga el profesor en sus labores docentes, juega un rol } \\
\text { importante, por cuanto se considera que, si este tiene más experiencia en su labor, } \\
\text { entonces gestionará de mejor forma su tiempo en el aula: “...al tener un poco más de } \\
\text { experiencia de cómo trabajan, del ritmo de los alumnos, uno empieza a pensar las } \\
\text { cosas distinto o a ser más efectivo” (Profesor enseñanza media, menos de } 15 \text { años de } \\
\text { experiencia). }\end{array}$ \\
\hline
\end{tabular}

Tabla 6: Categoría, Clima de Aula y Subcategoría, Beneficios del buen clima de aula.

\begin{tabular}{|c|c|c|}
\hline № & Teoría subjetiva & Comentario \\
\hline 1.1 & $\begin{array}{l}\text { Si existe un buen } \\
\text { clima de aula, } \\
\text { entonces se genera } \\
\text { un clima de } \\
\text { aprendizaje } \\
\text { adecuado. }\end{array}$ & $\begin{array}{l}\text { Para generar un ambiente de aprendizaje, primero se debe velar porque el clima de } \\
\text { aula sea el adecuado durante toda la clase o la mayor parte de ella, en cuanto se } \\
\text { plantea que para iniciarla se debe mantener la disciplina y el orden. Los docentes } \\
\text { consideran que no se debe continuar una clase con un mal clima de aula, sino que se } \\
\text { debe hacer una pausa para ordenar al curso o para retomar la atención de los } \\
\text { estudiantes y luego, continuar con el curso normal de la clase: “...la cuestión es } \\
\text { simple tu no podis tener un curso totalmente desordenado si vay a hacer clase, } \\
\text { entonces por lo menos teni que tener un ambiente adecuado para que tu podai hacer } \\
\text { tu clase, entonces en ese sentido quizás hay normas que no podi dejar pasar, aunque } \\
\text { el curso sea demasiado complicado y hay que trabajar mucho en eso” (Profesor } \\
\text { enseñanza media, menos de } 15 \text { años de experiencia). }\end{array}$ \\
\hline 1.2 & $\begin{array}{l}\text { Si existe un buen } \\
\text { clima de aula, } \\
\text { entonces se puede } \\
\text { ajustar el tiempo para } \\
\text { hacer } \\
\text { adecuadamente una } \\
\text { clase. }\end{array}$ & $\begin{array}{l}\text { Los docentes consideran que cuando existe un buen clima de aula, estos pueden } \\
\text { desarrollar efectivamente su labor, entonces los procesos de enseñanza y aprendizaje } \\
\text { se favorecen, en cuanto los estudiantes respetan al docente y al espacio pedagógico, } \\
\text { viéndose como necesidad la capacidad de los estudiantes de "autorregular su } \\
\text { comportamiento" para permitir al docente desarrollar adecuadamente la clase: “... } \\
\text { sea, si yo veo que mis chicos se autorregulan de una muy buena manera... } \\
\text { claramente tú puedes hacer una clase maravillosa, espectacular, donde se ajusten tus } \\
\text { tiempos" (Profesor enseñanza básica, menos de } 15 \text { años de experiencia). }\end{array}$ \\
\hline 1.3 & $\begin{array}{l}\text { Si existe un buen } \\
\text { clima de aula, el } \\
\text { tiempo fluye } \\
\text { adecuadamente. }\end{array}$ & $\begin{array}{l}\text { Los docentes consideran que, si existe un buen clima de aula, entonces el tiempo en } \\
\text { el aula "fluye". Esta fluidez del tiempo, es percibida por los docentes como si la clase } \\
\text { fuese más fácil de hacer, existiendo pocas interrupciones en el curso de la clase: "Hay } \\
\text { cursos que por hora o por capacidades intrínsecas también... o disposición también } \\
\text { intrínseca que tengan los cursos, es más fácil poder hacer una clase, por lo tanto, eso } \\
\text { genera que el tiempo se te haga más fluido" (Profesor enseñanza media, menos de } \\
15 \text { años de experiencia). }\end{array}$ \\
\hline 1.4 & $\begin{array}{l}\text { Si existe un buen } \\
\text { clima de aula el } \\
\text { tiempo se optimiza y } \\
\text { se percibe más } \\
\text { rápido. }\end{array}$ & $\begin{array}{l}\text { Los docentes consideran que, si se cumplen aquellos factores que benefician una } \\
\text { buena gestión del clima, la percepción que tienen del paso del tiempo es más rápida. } \\
\text { Esto quiere decir que si existe un buen clima de aula, expresado en disciplina y orden, } \\
\text { respeto por la clase y el docente, autorregulación del comportamiento por parte de los } \\
\text { estudiantes y disposición al aprendizaje, entonces la clase tendrá una estructura más } \\
\text { fluida y se aprovechará mejor el tiempo disponible para la instrucción académica. Por } \\
\text { lo tanto, la optimización y el buen uso del tiempo para las distintas actividades de } \\
\text { clase, permitiría percibir el paso del tiempo más rápido y fluido. } \\
\text { El mismo fenómeno ocurre a la inversa, si no existe un adecuado clima de aula y el } \\
\text { curso no tiene una actitud adecuada para el proceso de enseñanza-aprendizaje, el } \\
\text { trabajo en aula será más difícil para el docente y el tiempo pasará más lento: “...hay } \\
\text { cursos en donde el tiempo se hace más provechoso, se hace más rápido, porque los } \\
3 \text { momentos de la clase son mucho más fluidos, el inicio, el desarrollo y el cierre se } \\
\text { pueden hacer al menos correctamente. En cambio, en otros cursos donde son más } \\
\text { complicados, ... el tiempo se hace un poco más lento” (Profesor enseñanza media, } \\
\text { menos de } 15 \text { años de experiencia). }\end{array}$ \\
\hline
\end{tabular}


Tabla 7: Categoría Clima de Aula y Subcategoría, Perjuicios del mal clima de aula.

\begin{tabular}{|l|l|l|}
\hline № & Teoría subjetiva & Comentario \\
\hline 2.1 & $\begin{array}{l}\text { Si existe un mal clima } \\
\text { de aula, los } \\
\text { estudiantes no } \\
\text { lograrán los objetivos } \\
\text { de aprendizaje. }\end{array}$ & $\begin{array}{l}\text { Los docentes consideran que, si existe un mal clima de aula los estudiantes no logran } \\
\text { cumplir con los objetivos de aprendizajes, debido a las distracciones que genera el } \\
\text { mal comportamiento de los alumnos. Además, el desorden en aula, es un factor que } \\
\text { incide tanto en la capacidad atencional y de trabajo de los estudiantes, como en la } \\
\text { capacidad del docente para entregar los conocimientos e instrucciones de trabajo con } \\
\text { el fin de lograr los resultados esperados: "y también el clima dentro del aula, los } \\
\text { alumnos en general, si el curso no está con buen comportamiento tampoco van a } \\
\text { aprender, también se les va a dificultar" (Profesor enseñanza básica, menos de 15 } \\
\text { años de experiencia). }\end{array}$ \\
\hline 2.2 & $\begin{array}{l}\text { Si existe un mal clima } \\
\text { de aula, se perderá } \\
\text { tiempo de instrucción } \\
\text { con el fin de regular } \\
\text { el clima de aula. }\end{array}$ & $\begin{array}{l}\text { Generar estas intervenciones para regular el clima de aula, lleva a que los docentes } \\
\text { tengan menos tiempo para la instrucción académica, puesto que deben destinar } \\
\text { tiempo de su clase para regular el clima de aula necesario para comenzar con la } \\
\text { clase. La necesidad vista por los docentes por tener que generar un ambiente } \\
\text { adecuado, se debe a que, si no se establece un mínimo de orden y disciplina en el } \\
\text { aula, "no se podrá hacer clases", y en general se tiende a perder más tiempo: } \\
\text { “..Nosotros tenemos que ir a buscar alumnos al patio, porque no quieren entrar. } \\
\text { Entonces uno igual pierde tiempo en esas cosas, el hecho de pasar la lista igual es un } \\
\text { problema, el ruido afecta y uno se demora más en pasar los contenidos. Entonces } \\
\text { uno igual está jugando a contrareloj, porque quiero que aprendan valores y también } \\
\text { que aprendan contenidos. Y los tiempos de clase, efectivas, efectivas, se empiezan a } \\
\text { reducir" (Profesor enseñanza media, menos de 15 años de experiencia). }\end{array}$ \\
\hline
\end{tabular}

Tabla 8: Categoría Clima de Aula y Subcategoría, Gestión del clima de aula.

\begin{tabular}{|c|c|c|}
\hline № & Teoría subjetiva & Comentario \\
\hline 3.1 & $\begin{array}{l}\text { Si se gestiona el } \\
\text { clima de aula, } \\
\text { entonces este } \\
\text { mejorará y ayudará a } \\
\text { alcanzar los objetivos } \\
\text { de aprendizaje. }\end{array}$ & $\begin{array}{l}\text { Los docentes gestionan el clima de aula en sus clases, puesto que consideran que } \\
\text { sus acciones impactan positivamente en la convivencia escolar del curso y el clima de } \\
\text { este durante las clases, lo que beneficia al desarrollo adecuado de la instrucción } \\
\text { académica. Tienen TS según las cuales realizar acciones o actividades para influir en } \\
\text { el clima de aula tiene directa relación en cómo se desarrolla la clase, y por ende } \\
\text { podría ayudar a que se cumplan los objetivos de esta: "Hay muchos que no tienen } \\
\text { esas ganas de superarse, como que tienen una frustración y ya, se acabó, en cambio } \\
\text { las actividades deportivas y de convivencia, siento que son un apoyo grande, porque } \\
\text { son sus pares los que les dicen, "ya dale", "no es tan difícil", "yo te puedo ayudar", } \\
\text { entonces todo eso va mejorando el ambiente y ayuda en clases" (Profesor enseñanza } \\
\text { media, menos de } 15 \text { años de experiencia). }\end{array}$ \\
\hline 3.2 & $\begin{array}{l}\text { Si se gestiona el } \\
\text { clima de aula, } \\
\text { entonces mejorará el } \\
\text { rendimiento y el } \\
\text { comportamiento de } \\
\text { los estudiantes. }\end{array}$ & $\begin{array}{l}\text { Los profesores consideran que, si gestionan el clima de aula de los cursos, estos } \\
\text { tienen una recepción positiva y agradecen la intervención de los docentes. Esta } \\
\text { gestión, además de mejorar el comportamiento en el aula y promover una buena } \\
\text { convivencia escolar, permite mejorar también el rendimiento de los estudiantes, por } \\
\text { cuanto el docente comienza a conocer a los estudiantes y cómo estos aprenden, por } \\
\text { lo tanto pueden mejorar en la entrega de estos aprendizajes en función de las } \\
\text { características de los alumnos: "Yo creo que hoy en día al profesor le falta bastante } \\
\text { tiempo en ese sentido, a veces no es tan sólo importante el contenido, sino lo que } \\
\text { viene de trasfondo en cada comportamiento, sino también conocerlo, uno sabe cómo } \\
\text { abordarlo y uno sabe cómo llegar a él, como tranquilizarlo a todos... el curso lo tengo } \\
\text { ahora en cuarto medio y llevan, este sería el cuarto año siendo el mejor curso del } \\
\text { liceo, siendo en notas como en comportamiento... entonces es un cambio radical, de } \\
\text { alguna manera le estás dando tiempo..." (Profesor enseñanza media, menos de } 15 \\
\text { años de experiencia). }\end{array}$ \\
\hline
\end{tabular}

Derivado del modelo interpretativo (Figura 1), que organiza la TS general de los profesores entrevistados, se establece que la gestión del tiempo que realiza el docente se ve influida por obstaculizadores y facilitadores; del primero derivan características del estudiante, donde si no respetan al docente, se distraen en clases o tienen mala disposición al trabajo, los profesores tendrán más dificultades para gestionar el tiempo en aula. Además, si estos presentan conductas asociadas a carencias afectivas o problemas personales y familiares, el docente en su rol formador, tenderá a utilizar tiempo de su clase para apoyar en dicho ámbito a este grupo de estudiantes, en desmedro del tiempo disponible para la instrucción. Por otro lado, como parte de las características del docente que dificultan la gestión del tiempo, se encuentra el que el docente tenga una jefatura a su cargo, puesto que derivado de este rol, el profesor debe realizar una labor adicional que quitaría tiempo en su planificación de clases o incluso dejaría de realizar ciertas labores para atender a problemáticas emergentes, generalmente asociadas a problemas de convivencia. Por último, se encuentran características propias de la administración de los establecimientos, las cuales obstaculizan la gestión del tiempo del docente, 
entre estos se encuentran los problemas derivados de la convivencia escolar, excesiva cantidad de alumnos por curso y la diversidad de estudiantes, con lo cual el docente debe desplegar una mayor cantidad de estrategias en su práctica pedagógica, con el fin de favorecer el aprendizaje, que a criterio de los docentes, muchas veces no es suficiente.

Por otra parte, existen facilitadores que permiten una mejor gestión del tiempo en aula, los cuales son derivados de las características del estudiante y del docente; con respecto al primero, se encuentran como facilitadores el buen comportamiento, aludiendo a una buena convivencia en aula, y la disciplina, vista como una característica que permite la autorregulación del comportamiento esperado para el proceso de enseñanza aprendizaje, vinculada también a una buena disposición hacia el aprendizaje. Por parte del docente, se ha encontrado, que, si el docente tiene una relación cercana con sus estudiantes, puede monitorear su práctica pedagógica y ajustar sus intervenciones en aula, en relación a las expectativas e intereses de los estudiantes, además si el profesor tiene experiencia en su rol y se muestra comprometido ("vocación"), tenderá a gestionar más recursos personales para favorecer la gestión del tiempo en aula.

Dentro del esquema interpretativo (Figura 1), se observa el clima de aula como un factor que interviene en la gestión del tiempo, pudiendo manifestarse como un facilitador o un obstaculizador, en caso que exista buen clima o mal clima de aula, respectivamente. Cuando existe un clima negativo, la gestión del tiempo se ve perjudicada, además dificulta al proceso de enseñanza-aprendizaje y obliga al docente a destinar más tiempo de su clase para corregir conductas inapropiadas dentro del aula. Por lo tanto, hace que el docente pierda más tiempo, destinando tiempo de instrucción a disciplinar al curso para poder iniciar o continuar con la clase. Por otro lado, funcionando como un facilitador para la gestión del tiempo, el clima de aula positivo también favorece el aprendizaje, permite una mayor fluidez de la clase, existiendo menos interrupciones y situaciones que puedan detener el curso planificado de la instrucción académica. Finalmente, cuando existe un clima de aula positivo, la percepción del tiempo de los docentes es más acelerada, es decir, que los docentes al desenvolverse pedagógicamente en un buen clima de aula, perciben que el tiempo pasa mucho más rápido, por el contrario, cuando existe un clima inadecuado, los docentes perciben que el tiempo pasa más lento y lo refieren como "si el tiempo no avanzara".

Además, si el clima de aula se gestiona adecuadamente, este puede afectar los procesos de enseñanza aprendizaje, viéndose que cuando se realizan actividades a favor de la buena convivencia en el aula, considerada como aquella en la que existen relaciones de confianza y respeto, existe una adecuada disposición hacia el aprendizaje, y los estudiantes cumplen con los requisitos de disciplina y orden para realizar una clase. Cumpliéndose lo anterior el rendimiento de los estudiantes tendería a subir, el comportamiento en aula tendería a mejorar, y por último, el clima de aula mejoraría, favoreciendo el trabajo pedagógico y la gestión del tiempo en aula.

\section{DISCUSIÓN}

Sobre las TS de gestión del tiempo instruccional por parte de los docentes, se observó que la diversificación y la cantidad de estudiantes por sala es un factor que dificulta dicha gestión. Estas TS sustentan la visión problemática del sistema educativo chileno, en cuanto se tiende a homogeneizar la gestión del TI, pasando por alto el respeto por los ritmos y modos de aprendizaje (Cabrera y Herrera, 2016). Viéndose la gestión del TI como un recurso que permite aprovechar la mayor cantidad de tiempo para la instrucción, más que la calidad de esta en un tiempo determinado. Estos hallazgos ponen en evidencia la necesidad de reenfocar la discusión, en la calidad más que la cantidad del TI, en donde actualmente se da un mayor énfasis a la cultura escolarizante, más que la cultura centrada en el aprendizaje. En este sentido, un estudio realizado por Razo (2016), encontró que la extensión horaria de instrucción académica, no mejora los aprendizajes si el tiempo no es utilizado en interacciones de calidad entre estudiantes y docentes. Por el contrario, si este periodo es poco efectivo, pasar tiempo en la escuela podría complicar más aún la práctica docente, por lo tanto, el tiempo escolar es importante, pero aprovecharlo es fundamental, lo cual se podría lograr a través de una adecuada gestión del TI.

Al analizar los resultados en su contexto, se evidencia que en los establecimientos educacionales de grupo socioeconómico bajo, en su mayoría establecimientos de dependencia municipal (Ascorra et al., 2016; Martinic, 2015), se perciben los problemas de convivencia escolar y clima de aula, como uno de los elementos que más repercuten en el proceso de enseñanza aprendizaje (Wang y Degol, 2016). Lo anterior es coherente con lo encontrado en el presente estudio, con respecto a las dificultades que los docentes tienen para gestionar el tiempo de instrucción a causa de dificultades en el clima de aula.

Los docentes tienen TS acerca de que el clima de aula afecta el proceso de enseñanza-aprendizaje y el rendimiento académico, lo cual coincide con lo reportado por Ascorra et al. (2016), dando cuenta que los índices de convivencia escolar bajos, se encuentran en los mismos establecimientos que cuentan con un bajo rendimiento en la prueba PISA. De forma inversa, los establecimientos con mejores rendimientos académicos, presentarían un mejor índice de clima escolar. 


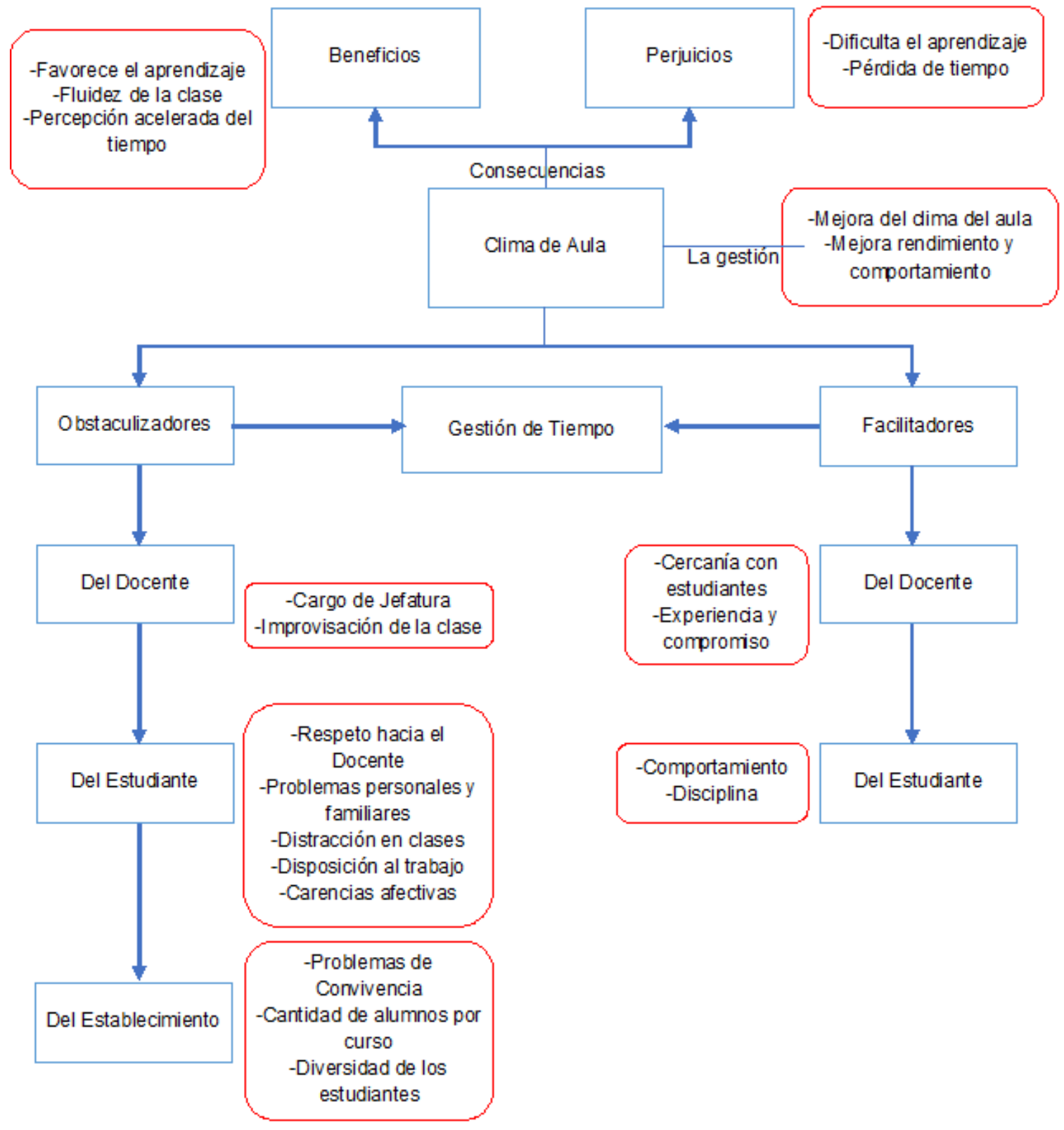

Fig. 1: Esquema general del modelo interpretativo de los resultados

Este fenómeno es explicado como un resultado de la segregación educativa que se genera a través de la selección de estudiantes, tanto en términos académicos como conductuales (Ascorra et al., 2016). Así mismo, los docentes refieren que las situaciones problemáticas más frecuentes corresponderían a dificultades en el clima de aula, específicamente aludiendo a conductas disruptivas en el aula, tales como, interrupciones, ruidos molestos, indisciplina, conversaciones fuera del contexto de aprendizaje, desorden, etc. (Córdoba et al., 2016; Nail et al., 2012). En este contexto de aprendizaje, y considerando su rol como gestores del clima de aula, los docentes se ven en la necesidad de intervenir cuando existen problemas de clima, antes de continuar con los contenidos curriculares, coincidiendo con lo hallado por Retuert y Castro (2017) en un estudio de teorías subjetivas acerca del rol docente en la construcción de la convivencia escolar. Considerando el contexto de este grupo de establecimientos, con índices de clima escolar inferiores al resto de los establecimientos, resulta coherente que los docentes tomen la decisión de utilizar más tiempo de instrucción para solucionar o atender a este tipo de dificultades, para generar un balance entre las demandas contextuales e institucionales (Nicolini, 2012), favoreciendo de esta forma la resolución de la clase. Lo anterior pone de relieve la necesidad de apoyar la política educativa inclusiva, con el fin de acortar la brecha de segregación por rendimiento académico y conducta de los estudiantes al momento de ingresar a los establecimientos educacionales. 
Se concluye que las TS de los docentes de los grupos analizados, consensuan que el clima de aula es un factor vital para la adecuada realización de clases, en cuanto su influencia va a beneficiar o perjudicar la gestión del tiempo que realice el docente en sus actividades de instrucción. De lo anterior, se puede deducir que el modo en que los agentes educativos reconocen y comprenden el clima de aula, permite desarrollar explicaciones en cómo estos gestionan el clima. Sin embargo, dadas las condiciones de los establecimientos de grupo socioeconómico bajo (excesiva cantidad de alumnos en sala de clases, conductas disruptivas, problemas personales y familiares de estudiantes, escaso compromiso/presencia de padres y apoderados, carencias afectivas, mal comportamiento e indisciplina), los docentes se ven frente a un ambiente de trabajo que no es el ideal para la instrucción, y por lo tanto deben gestionar los climas de aula de sus cursos, sin tener las herramientas o la experiencia suficiente para asegurar una adecuada gestión del mismo. Por lo tanto, conociendo los índices de clima de aula en estos establecimientos, que son los más bajos del país (Ascorra et al., 2016), surge como necesidad desarrollar políticas que promuevan una buena gestión de la convivencia escolar, a través de la capacitación de docentes en temáticas de gestión y organización de las aulas, como una forma de mejorar el comportamiento de los estudiantes, ordenar los procesos de enseñanza y aprendizaje, y con ello mejorar el aprendizaje, rendimiento académico y la buena convivencia en aula.

De las implicancias derivadas de este estudio, se establece como un aporte teórico a la investigación sobre teorías subjetivas en educación, dada la importancia que tiene la gestión del clima de aula como una herramienta para el adecuado trabajo pedagógico de los docentes en aula (Back et al., 2016); esta permitiría influir positivamente en la gestión del tiempo instruccional, contribuyendo a tener las condiciones necesarias para el proceso de enseñanza aprendizaje, dándole un ritmo fluido al proceso de instrucción académica.

Por otro lado, las TS reconstruidas desde los docentes del grupo socioeconómico bajo, permiten que docentes que se desempeñen en condiciones similares, puedan tomar como punto de intervención el capacitarse en habilidades de gestión de aula (clima y tiempo), para favorecer el proceso de enseñanza y aprendizaje de sus propios estudiantes. Además, derivada de las TS reconstruidas con respecto a la diversificación de las aulas, resulta necesario que los docentes en todos los grupos socioeconómicos, posean herramientas que permitan mejorar la gestión del tiempo instruccional, para favorecer y respetar con ello, los ritmos y modos de aprendizaje de todos los estudiantes (Cabrera y Herrera, 2016). Se establece como necesaria la capacitación en todos los grupos socioeconómicos, puesto que la diversificación de las aulas se sustenta en la Ley General de Educación (№ 20.370/2009), y en el decreto № 83/2015 que promueve la diversificación de la enseñanza, permitiendo el progreso de aprendizajes en igualdad de oportunidades de todo el estudiantado (MINEDUC, 2015).

Para finalizar, se concluye que la relación entre la gestión del TI y el clima de aula, es una relación directa, en cuanto si el clima de aula mejora, la gestión del tiempo tenderá a mejorar de la misma forma. Sin embargo, no se puede explicitar una relación directa de causalidad, pues se ha encontrado que el clima de aula incide en otros factores o condiciones, de los cuales, estos facilitarían u obstaculizarían la gestión tiempo instruccional. Por lo tanto, la gestión del clima de aula permitiría contar con las condiciones que permitirían al docente poder desarrollar la gestión del TI de forma adecuada, así como también, controlar aquellas condiciones que la obstaculizan.

De las limitaciones del estudio, se puede decir que no se realizó una sesión grupal para contrastar los resultados con la perspectiva de los participantes. Se considera una limitación, por cuanto no permite discutir los resultados entre los participantes, como modo de confirmar ciertas TS o explicitar aquellas que se encontraban implícitas en el discurso de los docentes. La importancia radica en que al trabajar sobre TS, el proceso de modificación de estas es un proceso continuo que puede realizarse en esta etapa de la investigación. Por otro lado, con respecto a la muestra, las TS de los docentes, no pueden ser vinculadas únicamente al trabajo en establecimientos de grupo socioeconómico bajo, puesto que no se determina como criterio excluyente de la muestra, el que trabaje o haya trabajado en otro establecimiento educacional con otro grupo socioeconómico, por lo tanto los datos extraídos pueden no ser alusiones únicas al grupo socioeconómico bajo.

Con respecto a los desafíos de la investigación de TS en el contexto educativo, se requieren líneas investigativas que aporten desde el ámbito práctico (Cuadra-Martínez y Castro-Carrasco, 2018), buscando estudiar el desarrollo y transformaciones de estas TS, y cómo al transformarse, pueden impactar en mejorar las prácticas pedagógicas de los docentes en la gestión del clima de aula y del tiempo instruccional, con el fin de complementar el desafío que implica producir el cambio en educación (Cuadra, et al., 2018).

\section{CONCLUSIONES}

La comprensión de las TS de los docentes, permite concluir que, existen distintos factores que pueden afectar la gestión del tiempo instruccional, de estos existen dos que facilitan una mejor gestión: 1) comportamiento adecuado y la disciplina del estudiante; y 2) compromiso y cercanía por parte del docente. Por otro lado, 
existen tres obstaculizadores que dificultan una adecuada gestión del tiempo: 1) características del estudiante (escaso respeto hacia el profesor, inadecuada disposición al trabajo, problemas socioemocionales tanto personales como familiares); 2) del docente (exceso de responsabilidades al tener una jefatura de curso a su cargo e improvisación de la clase); y 3) características de los establecimientos de grupo socioeconómico bajo (excesiva cantidad de alumnos en sala, problemas de convivencia y diversidad de estudiantes en aula). Además, se reconstruye una TS relacionada al clima de aula, que funciona como un factor que beneficia o dificulta la gestión del tiempo, si este es un clima adecuado o inadecuado. Por último, se establece que la gestión del tiempo instruccional permite mejorar el clima de aula, el comportamiento y el rendimiento académico de los estudiantes.

\section{REFERENCIAS}

Álvarez, D. y Lobo, G., El tiempo, uso y abuso, https://doi.org/10.4067/S0718-07642020000200073, Inf. Tecnol., 31(2), 73-80.

Ascorra, P., López, V., y otros cuatro autores, Relación entre segregación y convivencia escolar en escuela públicas chilenas, https://doi.org/10.11144/Javeriana.upsy15-1.rsce, Univ Psychol., 15(1), 65-78 (2016).

Aronson, J., Zimmerman, J., y Carlos, L., Improving student achievement by extending school: is it just a matter of time?, 1-9, WestEd, San Francisco, CA, United States (1998).

Back, L.T., Polk, E., Keys C.B., y McMahon, S.D., Classroom management, school staff relations, school climate, and academic achievement: testing a model with urban high schools, https://doi.org/10.1007/s10984-016-9213-x, Learn Environ Res., 19, 397-410 (2016).

Bonilla, M., Castro-Carrasco, P. y Gómez, V., Cambio de teorías subjetivas docentes ante sus primeras experiencias con estudiantes con síndrome de Down, https://doi.org/10.4067/S0718-07642020000300051, Inf. Tecnol, 31(3), 51-64.

Boniwell, I., y Zimbardo, P., Balancing Time Perspective in Pursuit of Optimal Functioning; in Positive Psychology in Practice by P.A. Linley and S. Joseph, pp 165-178, John Wiley and Sons, Hoboken, New Jersey, United States (2004).

Cabrera, V., y Herrera P., Una Escuela Con Nuevos Ritmos: Percepciones Sobre El Uso Del Tiempo Escolar, https://doi.org/10.4151/07189729-Vol.55-Iss.1-Art.371, Perspect. Educ., 55(1), 20-37 (2016).

Catalán, J., Hacia la formulación de una teoría general de las teorías subjetivas, https://doi.org/10.5027/psicoperspectivasVol15-Issue1-fulltext-739, Psicoperspectivas, 15(1), 53-65 (2016).

Cohen, J., McCabe, E., y Michelli, N., School Climate: Research, Policy, Practice, and Teacher Education, ISSN: 14679620, Teach Coll Rec., 111(1), 180-213 (2009).

Córdoba, F., Del Rey, R., Casas, J.A., y Ortega, R., Valoración del alumnado de primaria sobre convivencia escolar: El valor de la red de iguales, https://doi.org/10.5027/psicoperspectivas-Vol15-Issue2-fulltext-760, Psicoperspectivas, 15(2), 78-89 (2016).

Cuadra-Martínez, D., y Castro-Carrasco, P., Cambio educativo: propuesta de un modelo de transformación de teorías subjetivas, https://doi.org/10.5027/psicoperspectivas-Vol15-Issue2-fulltext-76010.24265/liberabit.2018.v24n1.10, Revista Peruana de Psicología, 24(1), 147-164 (2018).

Cuadra-Martínez, D., Castro, P., Vystrčilová, P., y Jancic-Mogliacci, R., A review of research on teacher's subjective theories: Contributions to the study of teacher education, Psychol. Educ., 54(3),1-22 (2017).

Cuadra, D., Salgado, J., Lería, F., y Menares, N., Teorías subjetivas en docentes sobre el aprendizaje y desarrollo socioemocional: Un estudio de caso, https://doi.org/10.15517/revedu.v42i2.25659, Rev. Edu., 42, 250-271 (2018).

Flick, U., An introduction to qualitative research, 3rd Ed., Sage, London, United Kingdom (2006).

Gobierno de Chile- MINEDUC, Diversificación de la enseñanza. Decreto №83/2015. División de Educación General, unidad de currículum. Santiago de Chile, MINEDUC (2015).

Kayode, G., y Ayodele, B., Impacts of Teachers' Time Management on Secondary School Students' Academic Performance in Ekiti State, Nigeria, https://doi.org/10.11648/j.ijsedu.20150301.11, Int J Second Educ., 3(1), 1-7 (2015).

Khan, H.M.A., Farooqi, M.T.K., Khalil, A., y Faisal, I., Exploring relationship of time management with teachers' performance, ISSN: 0555-7747, Bull. Educ, Res., 38(2), 249-263 (2016).

Lopes, J., Silva, E., y otros tres autores, Teacher's classroom management behavior and students' classroom misbehavior: A study with 5ththrough 9th- grade students, https://doi.org/10.14204/ejrep.43.17075, Rev Electron Investig Psicoeduc Psicopedag., 15(3), 467-490 (2017).

Martinic, S., El tiempo y el aprendizaje escolar la experiencia de la extensión de la jornada escolar en Chile, https://doi.org/10.1590/S1413-24782015206110, Rev. Bras. Educ., 20(61), 479-499 (2015).

Martinic, S., Vergara, C., y Huepe, D., The use of time and interactions in the classroom. A case study in Chile, https://doi.org/10.1590/S0103-73072013000100009, Pro-Posições, 24(1), 123-135 (2013).

Nail, O., Gajardo, J., y Muñoz, M., La técnica de análisis de incidentes críticos: una herramienta para la reflexión sobre prácticas docentes en convivencia escolar, https://doi.org/10.5027/psicoperspectivas-Vol11-Issue2-fulltext-204, Psicoperspectivas, 11(2), 56-76 (2012). 
Nicolini, D., Practice theory, work, and organization: An introduction, Oxford University Press, Oxford, United Kingdom (2012).

Pérez, A., Ferrer, R., y García, E., Tiempo escolar y subjetividad: significaciones sobre la práctica docente en escuelas de tiempo completo, ISSN: 1405-6666, Rev Mex Inv Educ, 20(65), 507-527 (2015).

Razo, A.E., Tiempo de aprender. El aprovechamiento de los periodos en el aula, ISSN: 1405-6666, Rev Mex Inv Educ., 21(69), 611-639 (2016).

Retuert, G., y Castro, P., Teorías subjetivas de profesores acerca de su rol en la construcción de la convivencia escolar, https://doi.org/10.4067/S0718-65682017000100321, Polis, 16(46), 321-345 (2017).

Strasser, K., Lissi, M., y Silva, M., Gestión del tiempo en 12 salas chilenas de Kindergarten: recreo, colación y algo de instrucción, https://doi.org/10.4067/S0718-22282009000100008, Psykhe, 18(1), 85-96 (2009).

Strauss, A., y Corbin, J., Basics of qualitative research. Techniques and procedures for developing grounded theory, 2nd Ed., Sage, United States (1998).

Ugwulashi, C.S., Cost, time and quality implications in school administration in Nigeria, ISSN: 2223-9553, Acad Res Int., 3(2), 405-411 (2012).

Ugwulashi, C., Time Management: Essential Tool For Teaching and Learning Improvement in Challenging, Educ Res Int., 1(2), 61-68 (2013).

Wang, M.T., y Degol, J.L., School climate: A review of the construct, measurement, and impact on student outcomes, https://doi.org/10.1007/s10648-015-9319-1, Educ Psychol Rev., 28(2), 315-352 (2016). 